\title{
Uso de YouTube como herramienta educativa de apoyo a la docencia en termodinámica e ingeniería térmica
}

\author{
Ignacio Zabalza a , Begoña Peña, Belén Zalba, José María Marín
}

Universidad de Zaragoza - Departamento de Ingeniería Mecánica, 50018 Zaragoza, España. aizabal@unizar.es

\begin{abstract}
The increasing use of Web 2.0 applications (blogs, YouTube, wikis, etc.) in higher education is favoring the adoption of new teaching methodologies as a complement to traditional teaching. This allows greater interaction with students improving the teaching/learning process. This article presents the main activities and results of a teaching innovation project funded by the University of Zaragoza (UZ) within the Open Resources Program (PRAUZ_18_012) where videos are being developed to support teaching in several subjects in the field of thermodynamics and thermal engineering. The videos contain theoretical explanations and short application exercises, They are made from a presentation that incorporates audio with the teacher's explanations. The set of videos was published within a specific YouTube channel and has various links from the Moodle pages of each subject. The article analyzes the visualization statistics of the videos, as well as the degree of satisfaction of the students through a questionnaire. The results show a high degree satisfaction of students with the videos produced as well as an improvement in the learning outcomes.
\end{abstract}

Keywords: educational videos, teaching innovation, ICT, OER, active methodologies, enginnering education.

\section{Resumen}

El creciente uso de las aplicaciones de la Web 2.0 (blogs, YouTube, wikis, etc.) en la enseñanza superior está favoreciendo la adopción de nuevas metodologías docentes complementarias a la docencia tradicional que permiten una mayor interacción con los estudiantes y una mejora del proceso de enseñanza-aprendizaje. Este artículo presenta las principales actividades y resultados de un proyecto de innovación docente financiado por la Universidad de Zaragoza (UZ) dentro del Programa de Recursos en Abierto (PRAUZ_18_012) donde se están elaborando vídeos como apoyo a la docencia en varias asignaturas del campo de la termodinámica y la ingeniería térmica. Los videos contienen explicaciones teóricas y pequeños ejercicios de aplicación, y se basan en una presentación que incorpora audio con las explicaciones del profesor. El conjunto de videos ha sido publicado dentro de un canal específico de la plataforma YouTube y cuenta con diversos enlaces desde las páginas Moodle de cada asignatura. El artículo analiza las estadísticas de visualización de los vídeos, así como el grado de satisfacción de los estudiantes evaluado en base a un cuestionario. Los 
resultados muestran un alto grado de satisfacción de los estudiantes con los vídeos elaborados así como una mejora en los resultados del aprendizaje.

Palabras clave: videos docentes, innovación docente, TIC, REA, metodologías activas, enseñanza en ingeniería.

\section{Introducción}

En los últimos años el uso de las aplicaciones de la Web 2.0 (Hew y Cheung, 2013), entre las que cabe destacar YouTube, wikis, redes sociales, blogs, etc. se ha extendido en todos los ámbitos, incluyendo también la enseñanza universitaria. De todas estas aplicaciones, YouTube es la más utilizada en el ámbito educativo, siendo muy bien valorada por los estudiantes universitarios (Ahmad, 2018).

Diversos estudios confirman que el uso de YouTube en la enseñanza universitaria ofrece ventajas complementarias a la docencia tradicional. Entre las principales mejoras cabe destacar que se promueve una mayor involucración y motivación de los estudiantes, y que éstos entienden y recuerdan mejor los conceptos teóricos más complejos gracias a las explicaciones visuales contenidas en los vídeos (Torres-Ramírez, 2014). Asimismo la elaboración de vídeos por parte de los estudiantes contribuye a la mejora en la adquisición de competencias transversales, incrementando al mismo tiempo su rendimiento académico (Orús et al., 2016), (Uyulgan y Akkuzu, 2018). Por otra parte, el uso de vídeos alojados en YouTube promueve la búsqueda de videos similares entre los estudiantes, adquiriendo así el hábito de usar esta plataforma como un recurso educativo (Chtouki et al., 2012).

Según diversos autores, el uso de vídeos docentes facilita el aprendizaje autónomo, constituyendo así una herramienta útil aplicable en distintos métodos de enseñanzaaprendizaje como el modelo de clase inversa (Peña et al., 2018), (Usón et al., 2018) o el aprendizaje combinado (Cagliero et al.,2017).

En cualquier caso, para conseguir un aprendizaje efectivo es fundamental la revisión, filtrado y selección previa de los vídeos recomendados a los estudiantes, ya que la visualización de videos de baja calidad académica tiene un efecto contraproducente, lo que puede ocasionar ciertas reticencias entre los profesores hacia la incorporación de YouTube en sus actividades docentes (Almobarraz, 2018). En este proceso de filtrado es conveniente revisar, entre otros aspectos, la autoría del vídeo y las opiniones de los usuarios, ya que algunos estudios han observado correlaciones significativas entre el grado de utilidad y efectividad del video y dichos aspectos (Lee et al., 2018).

Otro punto conflictivo en el uso de vídeos es el riesgo de que los estudiantes puedan percibir que van a aprender más con la visualización de un vídeo que con la asistencia a clase o con la lectura de un tema determinado en un libro, por lo que el uso de vídeos no debería plantearse como un sustituto sino como un complemento a la docencia tradicional (Moghavvemi et al., 2018). Por tanto, más que preguntarse qué recurso es mejor para el aprendizaje, lo recomendable es la combinación de los distintos recursos: vídeos, libros y aula (Meehan y McCallig, 2018). Además, mediante esta combinación se puede paliar el 
tradicional problema de falta de tiempo del docente para impartir su asignatura (Fernández, 2011).

Actualmente existe una gran variedad de tipos de vídeos docentes en función de los objetivos pedagógicos y los resultados de aprendizaje que se desee conseguir. Así la grabación de animaciones es útil para explicar conceptos abstractos, la captura de pantalla es adecuada para explicar el uso de un software determinado, etc. (Hansch et al., 2015). A pesar de que las clases enteras grabadas han sido tradicionalmente el tipo de vídeo más habitual, los estudiantes prefieren visualizar generalmente vídeos cortos específicamente elaborados en vez de clases grabadas, que a menudo contienen una gran cantidad de información para este tipo de formato (Meseguer-Martinez et al., 2017). En la tabla 1 se muestra una clasificación básica de distintos tipos de vídeos elaborada a partir de Hansch et al. (2015).

Tabla 1. Tipos de vídeos docentes comúnmente utilizados (basada en Hansch et al., 2015)

\begin{tabular}{ll}
\hline Tipo & Subtipo \\
\hline \multirow{2}{*}{$\begin{array}{l}\text { Cabeza parlante } \\
\text { con/sin superposición } \\
\text { de texto }\end{array}$} & Grabación en estudio \\
\cline { 2 - 2 } & Grabación sobre pantalla verde \\
\cline { 2 - 2 } & Grabación mediante captura de cámara web \\
\hline $\begin{array}{l}\text { Presentación de } \\
\text { diapositivas }\end{array}$ & Captura de PowerPoint con voz en off del profesor \\
\cline { 2 - 2 } & $\begin{array}{l}\text { Captura de PowerPoint con superposición de vídeo del profesor } \\
\text { explicando }\end{array}$ \\
\hline $\begin{array}{l}\text { Tableta digitalizadora } \\
\text { o pizarra digital }\end{array}$ & Captura de tableta digitalizadora \\
\cline { 2 - 2 } Profesor y pizarra & Captura de pizarra blanca digital \\
\hline Papel sobre escritorio & Grabación del profesor explicando en una pizarra \\
\hline Captura de pantalla & Captura de pantalla con voz en off del profesor \\
\hline Animación & Grabación de animaciones con voz en off del profesor \\
\hline Clase/Seminario & Grabación de una clase del profesor en el aula o de un seminario \\
\hline Entrevista & Grabación de una entrevista a un experto externo \\
\hline $\begin{array}{l}\text { Grabación de un experimento en un laboratorio o del funcionamiento de } \\
\text { una instalación o un proceso }\end{array}$ \\
\hline
\end{tabular}




\section{Objetivos}

El objetivo principal de este artículo es exponer las principales actividades llevadas a cabo por un grupo de profesores de la Escuela de Ingeniería y Arquitectura de la Universidad de Zaragoza (UZ) para el desarrollo y gestión de un canal en la plataforma YouTube, concebido como repositorio de videos docentes que sirven de apoyo a la docencia en varias asignaturas afines del ámbito de la termodinámica y la ingeniería térmica. Asimismo se persigue analizar los hábitos de visualización y el grado de satisfacción de los estudiantes y profesores de estas asignaturas con el uso de los vídeos, cuantificar las posibles mejoras obtenidas en el proceso de enseñanza-aprendizaje e identificar posibles desventajas o inconvenientes.

\section{Desarrollo de la innovación}

A continuación se presenta de un modo detallado la secuencia de actividades desarrolladas en este trabajo de innovación docente.

En primer lugar, se definió un grupo de asignaturas del mismo área de conocimiento (ver figura 1) con competencias y resultados de aprendizaje afines, y se conformó el grupo de profesores participantes en el proyecto. Seguidamente se establecieron los temas y resultados de aprendizaje más relevantes de las asignaturas previamente seleccionadas, lo que permitió preparar un listado, donde se definió la temática, tipología y el enfoque de los vídeos docentes a elaborar, las asignaturas en las que se utilizaría cada vídeo, así como el profesor encargado de su preparación y una fecha prevista para la realización de cada vídeo.

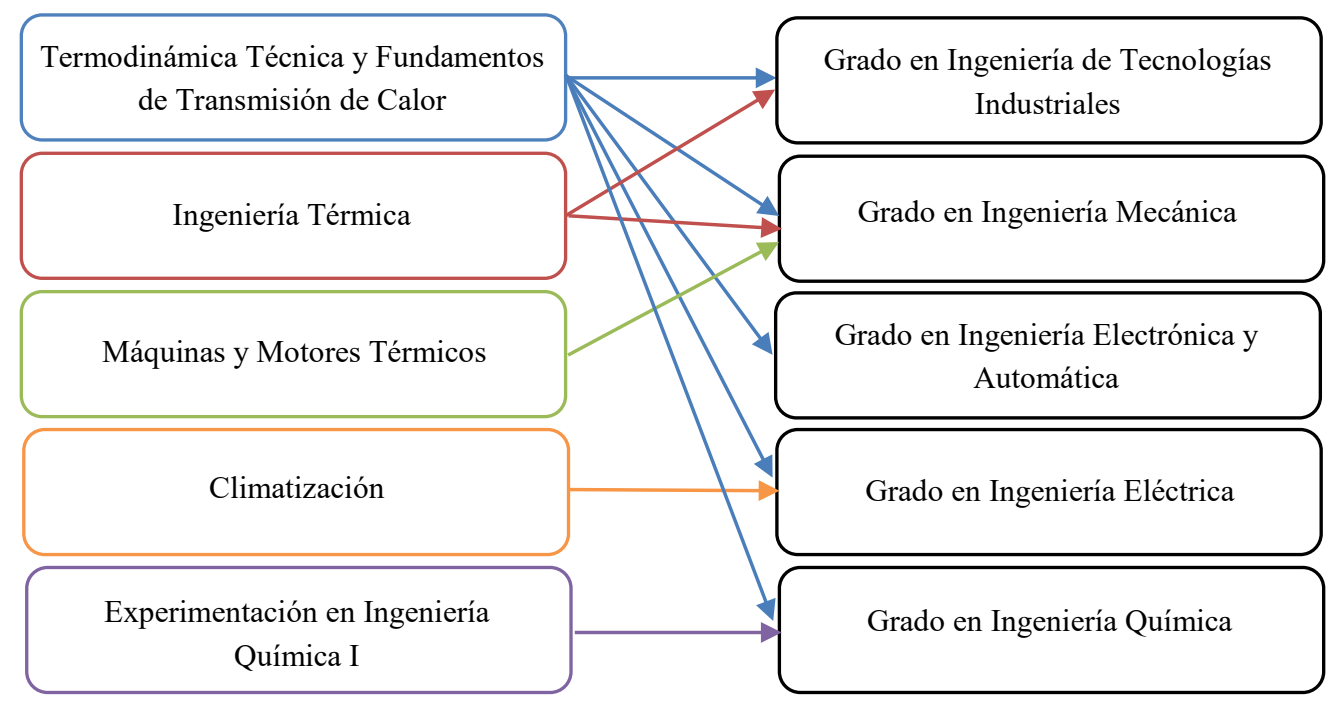

Fig. 1 Asignaturas de la UZ con competencias parcialmente cubiertas por los vídeos

Para este trabajo se decidió que la mayoría de los vídeos fueran del tipo "Presentación de diapositivas" que se basan en la captura de una presentación PowerPoint que incluye 
explicaciones teóricas y pequeños ejercicios prácticos de aplicación, incorporando la voz en off del profesor. No obstante, se han realizado también algunos vídeos (12\%) que explican el funcionamiento de distintos tipos de instalaciones de uso común (frigoríficos, sistemas de climatización para edificios, etc.) que aun estando basadas en la captura de una presentación PowerPoint incorporan también grabaciones de vídeo y/o fotografías de dichas instalaciones. La aplicación informática utilizada para la elaboración de todos los vídeos ha sido Camtasia, si bien en los próximos cursos se prevé realizar otras tipologías de vídeos basados en la resolución de problemas tipo mediante la captura de una tableta digitalizadora.

Cada vídeo docente elaborado pasó un proceso de revisión interna por parte del profesorado involucrado en el proyecto, como paso previo a su publicación en el canal "Innovación Docente Área MMT EINA-UZ" de la plataforma YouTube (ver figura 2), que fue creado unos meses antes de comenzar el actual curso académico 2018-19 con un número reducido de vídeos desarrollados en cursos anteriores.

En este punto, cabe señalar que todo el proceso de creación y gestión de un canal en YouTube es sencillo y requiere muy poco tiempo de dedicación. Para crear el canal, basta con crear previamente una cuenta en Google, seleccionar un nombre y una imagen de cabecera para el canal, incorporando también una breve descripción del mismo. Una vez creado, los vídeos pueden publicarse en el canal a medida que se van realizando, etiquetándolos correctamente con algunos metadatos, como: título, autoría, categoría, etc. Para conseguir una mayor eficiencia en todo este proceso, tan solo un profesor ha actuado como gestor del canal, quien se ha encargado de la publicación de los vídeos, la obtención y el seguimiento de las estadísticas de visualización por medio de "YouTube Analytics", la respuesta a los comentarios de los estudiantes, etc.

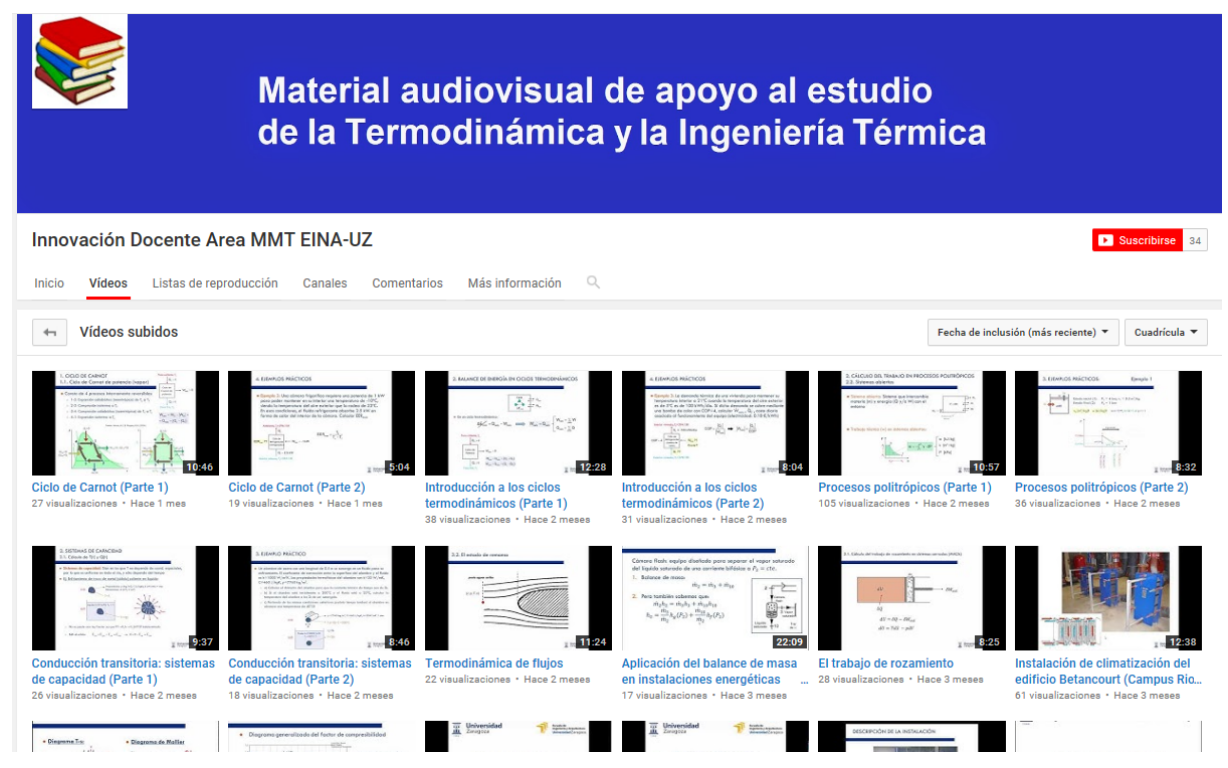

Fig. 2 Imagen del canal "Innovación Docente Área MMT EINA-UZ” en la plataforma YouTube 
Tras la publicación de cada vídeo en YouTube, el gestor del canal se ha encargado también de proporcionar la dirección URL de cada vídeo al resto de profesores participantes, de modo que éstos podían recomendar a sus estudiantes su visualización mediante la inserción de dicha dirección (a modo de link) en la página web interna de Moodle de la asignatura correspondiente.

Tras la finalización de cada semestre, los profesores realizaron una breve encuesta a los estudiantes para que indicasen su percepción y grado de satisfacción sobre la utilización de vídeos docentes en sus asignaturas. La encuesta se realizó a través de un cuestionario a través de Moodle o bien directamente en papel. Las preguntas incluidas en el cuestionario se muestran en la tabla 2.

Tabla 2. Cuestionario para evaluar el grado de satisfacción de los estudiantes

\begin{tabular}{ll}
\hline $\mathbf{N}^{\mathbf{0}}$ & Pregunta \\
\hline P1 & ¿Te interesan los vídeos? ( SI / NO ) \\
\hline P2 & ¿Cuántos vídeos has visto? ( Ninguno / Pocos / Bastantes / NS/NC ) \\
\hline P3 & ¿Te han parecido útiles los vídeos? ( SI / NO ) \\
\hline P4 & ¿Merece la pena seguir haciendo videos? ( SI / NO ) \\
\hline P5 & ¿Te gustaría que se elaborasen videos con problemas resueltos? ( SI / NO ) \\
\hline
\end{tabular}

\section{Resultados}

En esta sección se presentan en primer lugar las estadísticas generales de visualización de los vídeos del canal "Innovación Docente Área MMT EINA-UZ” con objeto de analizar los hábitos de visualización de los vídeos publicados. Conviene remarcar que dichas estadísticas no sólo incluyen a los estudiantes matriculados en las asignaturas participantes en el proyecto, sino a cualquier usuario de YouTube de cualquier lugar del mundo interesado en la temática abordada.

Posteriormente, a modo de ejemplo, se analiza el grado de satisfacción de los estudiantes y profesores de una de las asignaturas del proyecto. En concreto, se trata de la asignatura "Termodinámica técnica y fundamentos de transmisión de calor" del Grado en Ingeniería Eléctrica, que se imparte en el segundo año de la titulación y cuenta con seis créditos ECTS. En el curso 2018-19 hubo 61 estudiantes matriculados en esta asignatura.

\subsection{Análisis de los hábitos de visualización}

Transcurridos nueve meses desde su creación, el canal de YouTube "Innovación Docente Área MMT EINA-UZ” ofrece un total de 34 vídeos que se han ido incorporando al canal de forma progresiva. El canal cuenta actualmente con 36 suscriptores y se han registrado un total de 3.679 visualizaciones, con un tiempo de visualización total de 8 días, 13 horas y 48 minutos. Esto supone una duración media de las visualizaciones de 3:21 minutos frente a 
una duración media de los vídeos de 11:56 minutos, lo que conlleva un porcentaje medio reproducido del $28 \%$ aproximadamente.

El dispositivo preferido por los usuarios para visualizar los vídeos es el ordenador (77\%), seguido a distancia del teléfono móvil (20\%), siendo minoritario el uso de tablets y televisores.

La interacción con los usuarios es de momento relativamente baja, habiéndose recibido 40 "Me gusta", 1 "No me gusta" y 4 comentarios. En 37 ocasiones, los usuarios han compartido algún vídeo del canal y en 69 ocasiones los usuarios han incluido algún vídeo del canal en sus listas de reproducción.

En cuanto a la localización geográfica de los usuarios, aproximadamente el $28 \%$ del tiempo de visualización y del número de visualizaciones corresponde a usuarios españoles. A cierta distancia se situarían otros países de habla hispana como Ecuador y México con un 4\% y un $5 \%$ de las visualizaciones respectivamente, y posteriormente hay varios países con porcentajes inferiores al 1\% como Perú, Colombia o Bolivia. En contraste con lo anterior, los usuarios de Ecuador son los que cuentan con un mayor porcentaje medio reproducido en los vídeos, llegando a visualizar por término medio el $61 \%$ de la duración total de los vídeos, seguidos de los usuarios de Colombia y Perú con porcentajes del $49 \%$ y del 36\% respectivamente. Todos ellos están por delante de los usuarios españoles, cuyo porcentaje de reproducción promedio se queda en el $27 \%$.

La evolución mensual del número de visualizaciones y del porcentaje medio reproducido presenta un comportamiento similar. Tal como se puede observar en la figura 3, ambas métricas presentan un crecimiento muy significativo durante los primeros meses del curso académico (octubre y noviembre). Por otra parte, se observan también aumentos cuando se acercan los periodos de examen (junio, septiembre y enero).

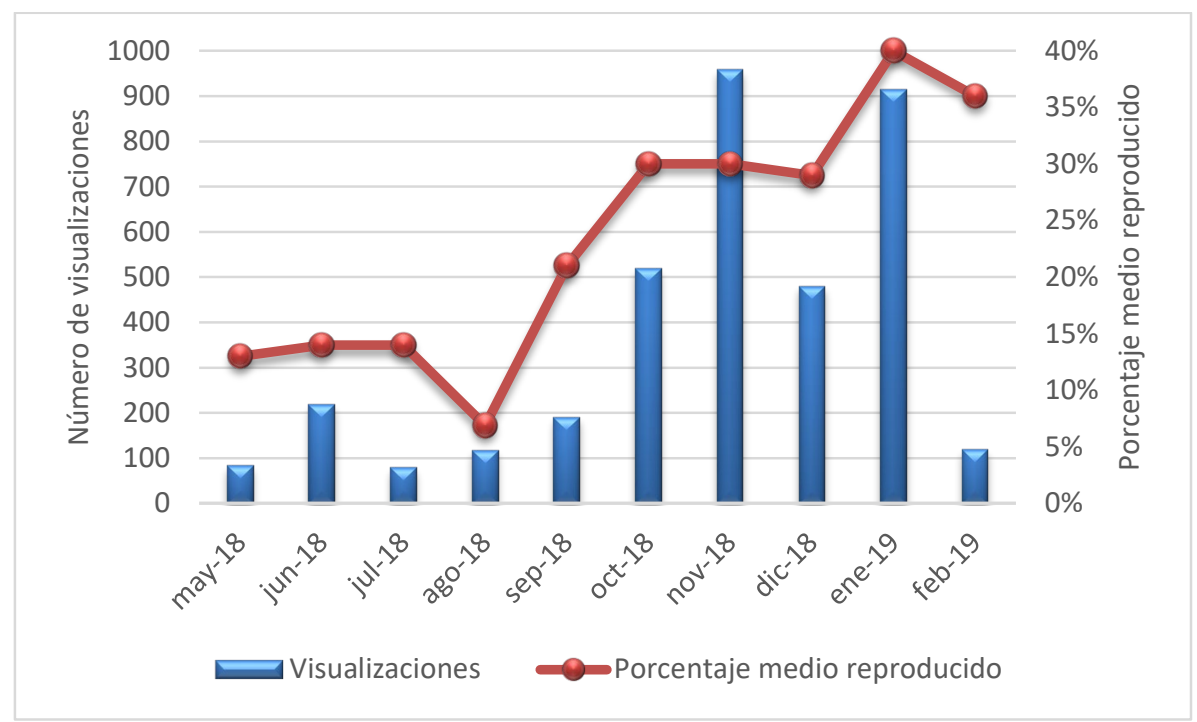

Fig. 3 Evolución mensual del número de visualizaciones y del porcentaje medio reproducido en el canal de YouTube "Innovación Docente Área MMT EINA-UZ" 
Uso de YouTube como herramienta educativa de apoyo a la docencia en termodinámica e ingeniería térmica

Analizando más detenidamente el tiempo de retención de los usuarios, al intentar establecer una correlación entre el porcentaje medio reproducido y la duración del vídeo, si bien puede apreciarse ligeramente que a mayor duración del vídeo, menor porcentaje reproducido del vídeo, no se observa una tendencia clara (ver figura 4), siendo necesario analizar periodos de tiempo más extensos para poder extraer conclusiones más precisas.

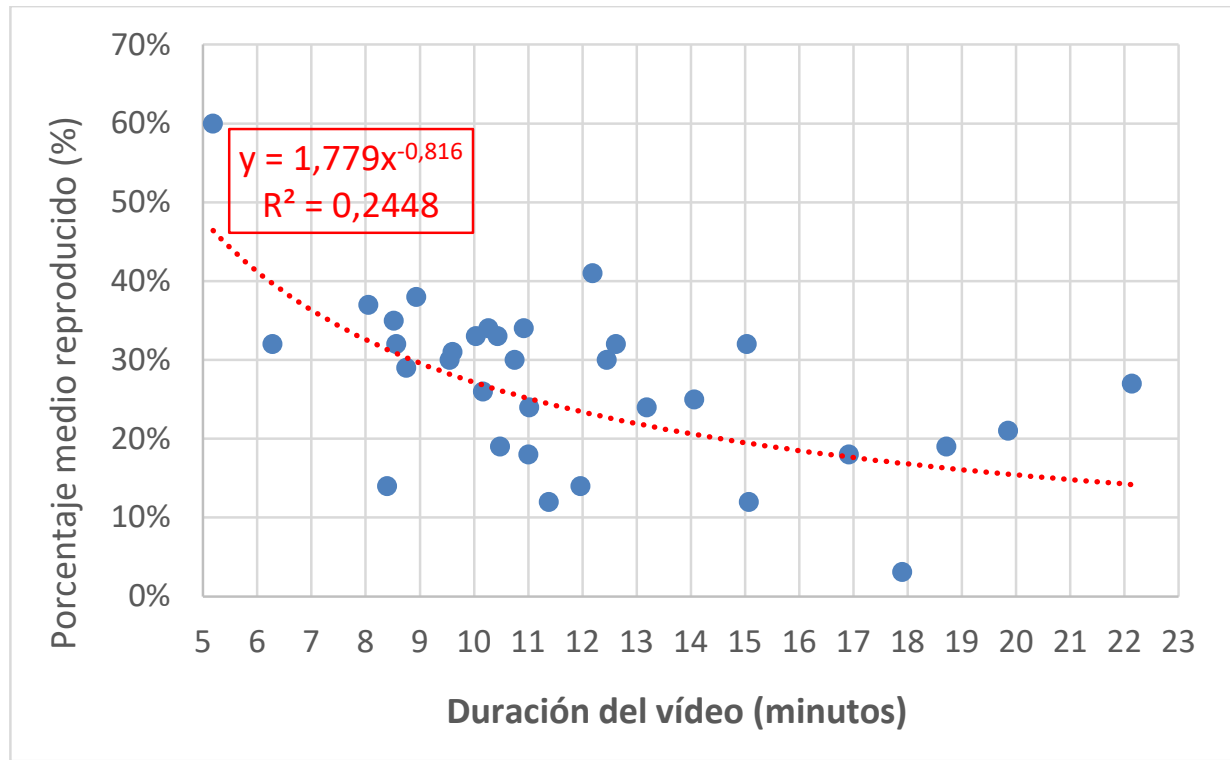

Fig. 4 Porcentaje medio reproducido en función de la duración de cada vídeo docente

En cualquier caso, los resultados están en consonancia con otros estudios similares que afirman que para garantizar una óptima retención de la audiencia la duración de los vídeos debería ser de 8 a 10 minutos (Ikastherm, 2019).

\subsection{Análisis del grado de satisfacción y de la mejora del aprendizaje de los estudiantes}

La participación de los estudiantes de la asignatura analizada en la encuesta de satisfacción sobre el uso de vídeos docentes fue voluntaria, respondiendo, no obstante, el 33\% de los estudiantes matriculados. Contrariamente a la percepción que puede tener el profesor acerca de la posible saturación de encuestas oficiales y no oficiales que se realiza a los estudiantes, en esta encuesta los estudiantes han apreciado de manera positiva que se tenga en cuenta su opinión.

En cuanto a los resultados de la encuesta, el $85 \%$ de los estudiantes afirma que le interesan los videos, aunque solamente un $35 \%$ afirma haber visto un número significativo de ellos. No obstante, al $80 \%$ les han parecido útiles y el $100 \%$ afirma que merece la pena que se sigan preparando y ofreciendo nuevos videos. En este sentido, cabe destacar que a la gran mayoría (90\%) les parece una buena idea que se elaborasen nuevos vídeos con problemas resueltos. 
Entre los comentarios realizados por los estudiantes en las encuestas cabe destacar la ventaja que para ellos supone poder visualizar los vídeos en el momento en que precisen y tantas veces como necesiten. De este modo, es posible adaptar el aprendizaje a su velocidad de comprensión al mismo tiempo que se les facilita una mayor flexibilidad en el tiempo dedicado al aprendizaje. Por otra parte, algunos estudiantes valoran positivamente la amenidad de los vídeos, por lo que puede ser un recurso de aprendizaje complementario a los recursos tradicionales como libros, clases magistrales, etc.

Por otra parte, los estudiantes de esta asignatura han valorado positivamente el interés del profesorado en la mejora de su aprendizaje mediante el uso de vídeos y otros recursos, propiciando así una mejora de los resultados de esta asignatura en las encuestas oficiales de evaluación de la actividad docente.

Finalmente, con respecto a las calificaciones, en este curso académico el $62 \%$ de los estudiantes matriculados se han presentado al examen de la asignatura en primera convocatoria, de los cuales el $68 \%$ lo han superado, lo que supone una mejoría con respecto a cursos anteriores en los que no se habían utilizado vídeos y esta cifra se situaba en el rango del $40-60 \%$.

\subsection{Análisis del grado de satisfacción de los profesores}

En opinión de los profesores participantes en este trabajo, además de lo que aportan los videos a los estudiantes, tienen un valor adicional para los profesores, permitiendo ver cómo explican otros profesores una determinada temática, lo que permite aprender unos de otros, discutir e intercambiar distintos enfoques, metodologías y puntos de vista, etc. Esto es especialmente interesante para los profesores que imparten una asignatura por primera vez.

En el caso particular de los videos sobre instalaciones utilizadas en prácticas y/o visitas cabe destacar su utilidad tanto para ayudar a los estudiantes a preparar la sesión de prácticas o la visita a la instalación correspondiente como para ayudar a los profesores a compartir conocimientos prácticos relativos al equipamiento docente disponible, que algunas veces puede no ser bien conocido por todos los profesores.

Si bien la realización de vídeos conlleva una cierta carga de trabajo para los profesores, ésta puede atenuarse mediante el desarrollo progresivo de estos materiales entre varios profesores y a lo largo de varios cursos académicos.

Finalmente, el hecho de poder disponer de un repositorio común de materiales docentes conlleva una mejor coordinación, además de un ahorro de espacio de almacenamiento en los servidores de la Universidad.

\section{Conclusiones}

A partir de los resultados de este trabajo se puede concluir que el uso de vídeos como material complementario a la docencia tradicional en el campo de la termodinámica y la 
ingeniería térmica es generalmente valorado de forma positiva tanto por los estudiantes como los profesores, produciendo una mejora en el aprendizaje que se traduce en unas mejores calificaciones finales. Además, esta conclusión coincide con lo ya apuntado en la mayor parte de las publicaciones sobre experiencias de innovación similares encontradas en la bibliografía.

A pesar de que las tecnologías requeridas son sencillas de utilizar y fáciles de adquirir, la elaboración de los vídeos docentes conlleva una cierta dedicación de tiempo por parte de los profesores que es preciso planificar de antemano.

Respecto a los hábitos de visualización de los vídeos, a pesar de que los resultados confirman algunas tendencias ya apuntadas por otros autores, como la conveniencia de hacer vídeos cortos (de entre 8 y 10 minutos), hay que remarcar que el canal de YouTube se creó hace tan solo nueve meses, por lo que se considera necesario ampliar el tiempo de observación y aumentar el número de visualizaciones para poder obtener resultados con mayor fiabilidad estadística.

Finalmente sería conveniente seguir trabajando para mejorar la retroalimentación entre estudiantes y profesores, con objeto de obtener más información sobre el modo de aprendizaje de los estudiantes y su valoración de los recursos utilizados, el tiempo requerido, su grado de motivación y expectativas, etc. Asimismo sería interesante disponer de resultados de diferentes asignaturas en las que se utilicen los mismos vídeos para poder realizar análisis comparativos.

\section{Agradecimientos}

Este trabajo ha sido financiado por medio del proyecto PRAUZ_18_012 perteneciente al Programa de Recursos en Abierto de la convocatoria 2018-19 de Proyectos de Innovación Docente del Vicerrectorado de Política Académica de la Universidad de Zaragoza. El proyecto ha sido coordinado por el Prof. Ignacio Zabalza Bribián, siendo los profesores participantes durante el curso 2018-19: Begoña Peña Pellicer, Eva $M^{a}$ Llera Sastresa, Sergio Usón Gil, Ma Belén Zalba Nonay, José María Marín Herrero, Luis María Serra de Renobales, Mariano Muñoz Rodríguez, Francisco Moreno Gómez, Francisco Javier Uche Marcuello, Ana Lázaro Fernández y Mónica Delgado Gracia. Agradecemos la contribución de todos los profesores y estudiantes que han participado en este proyecto.

\section{Referencias}

AHMAD, F. (2018). "YouTube as Engagement and Learning Tool in Higher Education Society" en Journal of Fundamental and Applied Sciences, vol. 10, issue 4S, pp. 137-142.

ALMOBARRAZ, A. (2018). "Utilization of YouTube as an information resource to support university courses" en Electronic Library, vol. 36, issue 1, pp. 71-81. 
CAGLIERO, L., FARINETTI, L., MEZZALAMA, M., VENUTO, E. y BARALIS, E. (2017). "Educational video services in universities: A systematic effectiveness analysis" En: FIE 2017 Frontiers in Education Conference Proceedings, Indianapolis: IEEE. 1-9.

CHTOUKI, Y., HARROUD, H., KHALIDI, M. y BENNANI, S. (2012). "The impact of YouTube videos on the student's learning" en ITHET 2012-International Conference on Information Technology Based Higher Education and Training. Disponible en $<$ https://ieeexplore.ieee.org/document/6246045> [Consulta: 31 de enero de 2019].

FERNÁNDEZ, PG. (2011) “Autonomous learning with audiovisual materials [Aprendizaje autónomo utilizando vídeos docentes]" en Revista Iberoamericana de Tecnologías del Aprendizaje, vol. 6, issue 2, pp. 65-69.

HANSCH, A., HILLERS, L., MCCONACHIE, K., NEWMAN, C., SCHILDHAUER, T. y SCHMIDT, P. (2015) "Video and Online Learning: Critical Reflections and Findings from the Field" en HIIG Discussion Paper Series, no. 2015-02. $<$ https://ssrn.com/abstract=2577882 $>$ [Consulta: 31 de enero de 2019].

HEW, K.F. y CHEUNG, W.S. (2013). "Use of Web 2.0 technologies in K-12 and higher education: The search for evidence-based practice" en Educational Research Review, vol. 9, pp. 47-64.

IKASTHERM. Active learning methodology based on legacy tools: flipped learining methodology. <https://www.youtube.com/watch?v=XOwodn1ukxs\&feature=youtu.be $>$ [Consulta: 31 de enero de 2019]

LEE, H., CHOI, A., JANG, Y. y LEE, J.I. (2018). "YouTube as a learning tool for four shoulder tests" en Primary Health Care Research and Development. Article in Press.

MEEHAN, M. y MCCALLIG, J. (2018). Effects on learning of time spent by university students attending lectures and/or watching online videos" en Journal of Computer Assisted Learning, Article in Press.

MESEGUER-MARTINEZ, A., ROS-GALVEZ, A. y ROSA-GARCIA, A. (2017). "Satisfaction with online teaching videos: A quantitative approach" en Innovations in Education and Teaching International, vol. 54, issue 1, pp. 62-67.

MOGHAVVEMI, S., SULAIMAN, A., JAAFAR, N.I. y KASEM, N. (2018). "Social media as a complementary learning tool for teaching and learning: The case of youtube" en International Journal of Management Education, vol. 16, issue 1, pp. 37-42.

ORÚS, C., BARLÉS, MJ., BELANCHE, D., CASALÓ, L., FRAJ, E. y GURREA, R. (2016) "The effects of learner-generated videos for YouTube on learning outcomes and satisfaction" en Computers and Education, vol. 95, pp. 254-269.

PEÑA, B., ZABALZA, I., LLERA, E.M., USÓN, S. y ROMEO, L.M. (2018). “Aplicación del modelo de clase inversa en el campo de la Ingeniería Térmica: análisis y comparación de varios contextos" en Congreso In-Red 2018, IV Congreso Nacional de Innovación Educativa y Docencia en Red. Valencia: Universitat Politècnica de València. 908-921. 
Uso de YouTube como herramienta educativa de apoyo a la docencia en termodinámica e ingeniería térmica

TORRES-RAMÍREZ, M., GARCÍA-DOMINGO, B., AGUILERA, J. y DE LA CASA, J. (2014). "Video-sharing educational tool applied to the teaching in renewable energy subjects" en Computers and Education, vol. 73, pp. 160-177.

USÓN, S., PEÑA, B., ZABALZA, I., LLERA, E.M. y ROMEO, L.M. (2018). “Combining Flipped Classroom Model and Educational Videos for Improving Teaching-Learning Process in Thermodynamics and Thermal Engineering" en Proceedings, vol. 2, issue 21, pp. 1-4.

UYULGAN, M.A. y AKKUZU, N. (2018). "Educational short videos to utilize in the biochemistry laboratory: Opinions of university students" en Journal of Baltic Science Education, vol. 17, issue 3, pp. 496-510. 